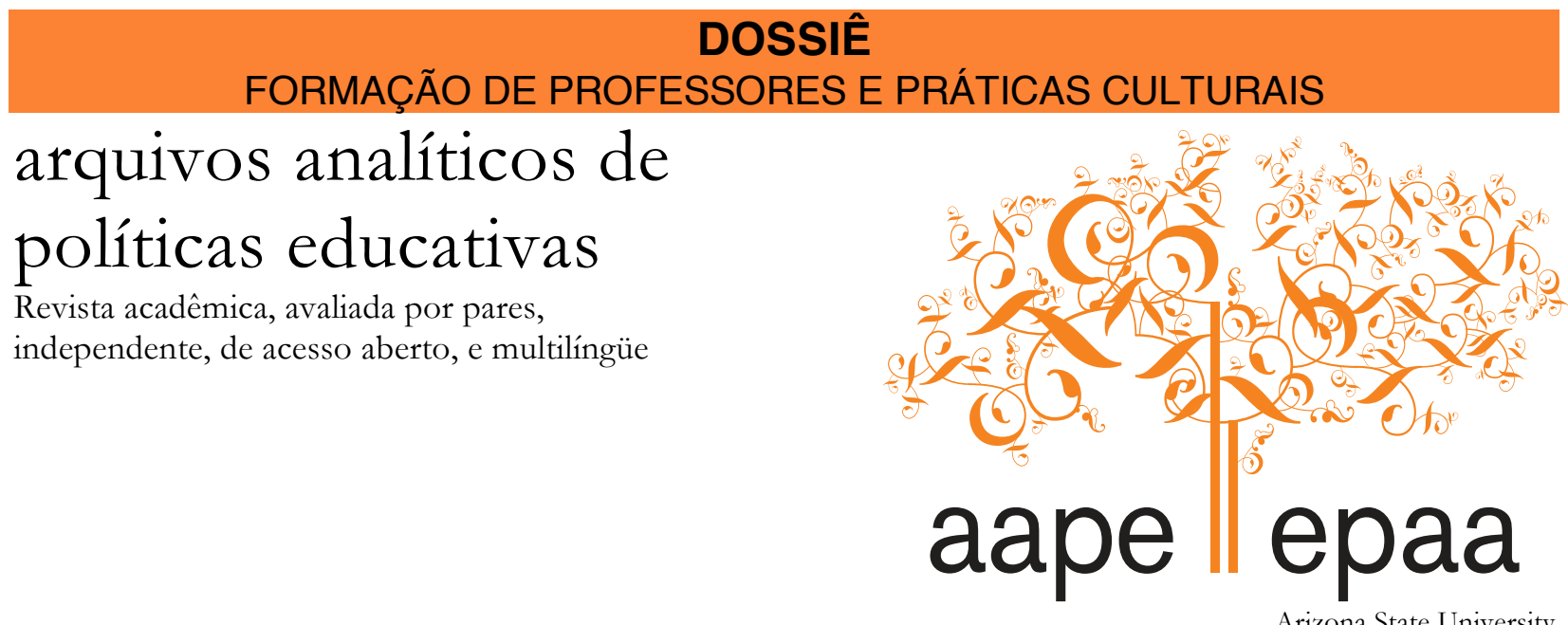

Arizona State University

Volume 21 Número $28 \quad 25$ de março, 2013

ISSN 1068-2341

\title{
La Experiencia Como Eje De La Formación: una propuesta de Formación Inicial de educadoras y educadores sociales
}

\author{
Nieves Blanco García \\ José Eduardo Sierra Nieto \\ Universidade de Málaga \\ España
}

Citação: Blanco García, N. y Sierra Nieto, J.E. (2013). La Experiencia Como Eje de la Formación: una propuesta de formación inicial de educadoras y educadores sociales. Arquivos Analíticos de Políticas Educativas, 21(28). Dossiê Formação de Professores e Práticas Culturais: descobertas, enlaces, experimentações. Editoras convidadas: Carla Beatriz Meinerz, Dóris Maria Luzzardi Fiss \& Sônia Mara Moreira Ogiba. Recuperado [data] http://epaa.asu.edu/ojs/article/view/1143

Resumen: El sentido de la formación inicial es ayudar a que las alumnas y los alumnos desarrollen sus modos propios de pensar, de actuar, explicitando y reflexionando sobre los principios que orientan sus acciones. Por tanto, consideramos que nuestro papel como enseñantes es favorecer que las y los estudiantes puedan formar-se, conocer-se, que desarrollen un saber vivo, encarnado, donde el saber, el hacer y el ser se presentan unidos. Desde estos presupuestos, desarrollamos una propuesta de formación que toma como base la experiencia: la de los estudiantes, la de profesionales en ejercicio, y la nuestra propia como docentes. En las páginas que siguen, abordamos las concepciones que la orientan, los principios y opciones metodológicas en que se concreta, y algunas reflexiones sobre los retos y posibilidades que se abren.

Palabras clave: Saber de la experiencia; Experiencia; Formación inicial; Relación de autoridad; Reflexión. 
Experience as the focus of development: A proposal for the training of social pedagogues. Abstract: The meaning of initial training is to help the students to develop their own ways of thinking, acting, explaining and reflecting on the principles that guide their actions. We therefore believe that our role as teachers is to encourage the students to develop their own ways of thinking and acting based on their own "lived's experiences". From these assumptions, we've develop a training proposal that builds on the experience: that of students: ours as teachers and that of professional educators. In the pages that follow, we discuss the concepts that guide ours principles and methodological options, and some reflections on the challenges and possibilities that open. Keywords: Knowing from experience; Experience; Initial Training; Pedagogical Relationship; Reflection.

\section{A Experiência Como Eixo da Formação: uma proposta de Formação Inicial de educadores e educadoras sociais}

Resumo: O sentido da Formação Inicial reside em ajudar alunos e alunas a desenvolverem modos próprios de pensar e atuar, explicitando e produzindo reflexões sobre os princípios que orientam suas ações. Portanto, consideramos que nosso papel, como ensinantes, é favorecer que os/as estudantes possam formar-se e conhecer-se, desenvolvendo um saber vivo, encarnado, a partir do qual o saber, o fazer e o ser estabeleçam relações entre si. A partir destes pressupostos, desenvolvemos uma proposta de formação que toma como base a experiência: a dos estudantes, a dos professores que estão atuando, a nossa própria experiência como docentes. Nas páginas que seguem, abordamos as concepções que orientam essa experiência, os princípios e opções metodológicas por meio das quais ela se materializa, produzindo algumas reflexões sobre os caminhos e possibilidades que se abrem.

Palavras-chave: Saber de experiência; Experiência; Formação Inicial; Relação de Autoridade; Reflexão.

\section{Introducción $^{1}$}

En este texto presentamos una propuesta de formación en el Grado de Educación Social en la Universidad de Málaga (España), que hemos comenzado a desarrollar en la asignatura de $2^{\circ}$ curso: Diseño, desarrollo, evaluación e innovación de proyectos de intervención socioeducativa ${ }^{2}$. Con esta propuesta tratamos de dar forma a nuestra concepción sobre el sentido de la Formación Inicial de Educadoras y Educadores Sociales ${ }^{3}$, centrando la atención en recrear saberes experienciales,

\footnotetext{
${ }^{1}$ Este texto se enmarca en el Proyecto de Investigación EDU2011-29732-C02-01, financiado por el MICINN para el periodo 2012-2014.

${ }^{2}$ Esta materia forma parte del Grado de Educación Social impartido en la Universidad de Málaga; titulación que comenzó su andadura en el curso académico 2010/2011 (anteriormente existía un plan de estudios distinto). Se trata de una asignatura Anual de $2^{\circ}$ año (de un total de 4), cuyo propósito general tiene que ver con el desarrollo de un conocimiento crítico de las bases teóricas y metodológicas que sustentan los procesos de diseño, desarrollo, evaluación e innovación de proyectos de intervención socioeducativos. Para más información acerca de la titulación, es posible consultar la Web de la Facultad de Educación de la Universidad de Málaga: http://www.cceducacion.t2v.com/titulacion/horario/id/14/curso/1

${ }^{3}$ La figura profesional del educador social en España, tal y como la entendemos en la actualidad, es el resultado de tres tradiciones educativas históricas diferentes: educadores especializados, animadores socioculturales y educadores de adultos (Sáez Carreras y Molina, 2006, p. 100). A través de la instauración de una titulación superior única (Diplomatura Universitaria en Educación Social, año 1991), se trató de aglutinar
} 
desempeñar un papel de mediadores, tratando de provocar la reflexión de las alumnas y los alumnos sobre sus vivencias; todo ello con el propósito de que puedan ir dando forma a sus propios saberes profesionales. Y, al hacerlo, también estamos recreando nuestros propios saberes como docentes, analizando nuestras decisiones, el modo en que las concretamos y el reflejo que nos devuelven las alumnas y los alumnos, como una forma de orientar la práctica de la evaluación educativa (Santos Guerra, 2002).

Para nosotros, la naturaleza de la acción socioeducativa requiere que la formación esté articulada en un diálogo vivo entre las tradiciones de pensamiento (las teorías) y la experiencia vivida de las y los estudiantes (Van Manen, 2003; Contreras y Pérez de Lara, 2010). Las situaciones y los problemas a los que habrán de enfrentarse como educadoras y educadores, los saberes relacionales que habrán de desplegar, no son un asunto técnico que se resuelva aplicando un conocimiento. Más bien, responde a lo que diferentes autores han denominado saberes prácticos ${ }^{4}$ (Contreras, 2010; Tardiff, 2004), y que en nuestro caso estamos trabajando a través de la conceptualización de Hannah Arendt (2002) sobre la actividad humana, y las implicaciones que esto tiene para la educación (Bárcena y Mèlich, 2000).

El eje principal sobre el que pivota la asignatura tiene que ver con la reflexión sobre el sentido educativo que orienta toda propuesta de acción socioeducativa, procurando desarrollar la capacidad de toma de consciencia acerca de los pilares sobre los que se asienta nuestro criterio profesional. El sentido de organizar así la materia tiene que ver con nuestra convicción acerca de que, como educadoras/es, nuestro papel no es gestionar o aplicar proyectos sino realizar un trabajo educativo a través de proyectos. Los programas o proyectos de intervención son, de acuerdo con estos planteamientos, vehículos a través de los que la educadora, el educador, se muestra, actúa, a menudo en una relación compleja y llena de paradojas y obstáculos, en la que - como señala Asunción López (2006, p. 140) - "quien no da sentido, lo recibe impuesto". El sentido de su acción vendrá dado por el programa, impuesto, sólo en el caso de que - como profesional - no sea capaz de orientarlo desde su propio criterio sobre el valor educativo de lo que hace, tomando una posición reflexiva y consciente en su diseño y desarrollo.

En las líneas que siguen, y como fruto de un proceso de investigación de auto-estudio ${ }^{5}$ (CochranSmith y Lytle, 2002), exponemos parte del trabajo que venimos desarrollando, a partir del que ha sido nuestro principal propósito: organizar la Formación Inicial tomando como base la experiencia, buscando favorecer que las y los estudiantes generen saberes propios reflexionando sobre lo que viven y dialogando con el saber de la experiencia de profesionales en ejercicio. Tomaremos algunas palabras de nuestros estudiantes para pensar en qué medida nuestros propósitos están siendo acogidos y pueden estar - o no - resultando valiosos para la formación de estas chicas y de estos chicos, y concluiremos con algunas reflexiones sobre los retos que plantea pensar - y hacer - de este modo la formación.

estas tradiciones en una sola (unido al esfuerzo profesionalizardor de los colegios y las asociaciones profesionales).

${ }^{4}$ Se denominan saberes prácticos o experienciales a los que desarrollan quienes se dedican a actividades educativas, que son necesarios para el ejercicio de su oficio y que se originan en la práctica cotidiana, en diálogo con la realidad que viven. Se trata, por tanto, de un saber propio y singular, que toma cuerpo (se encarna) en el educador, lo implica completamente, y es fruto de la experiencia vivida y de la reflexión sobre ella.

${ }^{5}$ Según estas autoras, la metodologia de auto-estudio consiste en indagaciones sobre la propia práctica (utilizando recursos propios de la investigación narrativa y la investigación-acción) y está asociada a profesorado universitario que trabaja en formación inicial. Tiene una extensa tradición, como muestra la existencia de un handbook específico (Loughran, 2004). 


\section{La Formación Inicial y el aprendizaje del oficio educativo}

Es patente que la formación universitaria está sostenida desde una cultura profesional concebida como una suma de aportaciones teóricas proveniente de diferentes campos de conocimiento; aportaciones que suelen presentarse de forma fragmentada y con poca posibilidad para establecer relaciones significativas por parte de las y los estudiantes. Para el campo de la educación, como nos recuerda Contreras (2010, p. 68), esta crítica general nos lleva a reconocer que "disciplinas como la Didáctica y la Pedagogía en general, son presentadas y concretadas a menudo como un discurso acerca del hacer educativo, pero desligado del propio hacer". Korthagen (2011) reconoce que la formación universitaria (se refiere especialmente a la Facultades de Educación, aunque es una crítica que se podría hacer extensible a otros centros de Formación Superior) se sigue basando en un modelo donde "la Universidad aporta la teoría y las escuelas [los centros de trabajo en un sentido más amplio] la oportunidad de poner dicha teoría en la práctica” (p. 56).

Aún más problemática es la situación, constatada a través de múltiples fuentes, del divorcio entre el conocimiento pedagógico y el saber generado en los contextos prácticos, en los lugares donde se genera el saber de la experiencia. Un saber que, en la formación universitaria, o bien está ausente o es despreciado (Blanco, 2010). Ello muestra, como ha constatado para la escuela Cristina Mecenero (2009), la pervivencia de la lógica jerárquica en la producción de conocimiento pedagógico (Contreras, 1991), y la falta de expectativas de la comunidad universitaria hacia un saber que, para ella, es necesario y suficiente para enseñar y aprender a enseñar.

Todo ello es especialmente alarmante si reparamos en que, con mucha frecuencia, cuando se hace recuento de los espacios donde hemos aprendido sobre nuestra profesión, la Universidad no es mencionada en primer lugar. Más bien suele indicarse que allí no aprendimos nada - o poco relevante.

Nuestros estudiantes viven estas contradicciones y las afrontan de diversos modos: algunos con disciplina y obcecación; otros con resistencias y actitud de crítica abierta; otros, entablando relaciones instrumentales con el trabajo que les proponemos. Frente al conocimiento teórico o, mejor, frente a las formas de mediación que les proponemos con la teoría (que resultan en demasiadas ocasiones formas muy instructivas), muestran predilección hacia "lo práctico", en el sentido de hacer'. Sin embargo, pensamos que la cuestión importante no es tanto una elección entre cuánta teoría y cuánta práctica, sino de cómo la enseñanza puede convertirse en una oportunidad para entrar en relación con distintas tradiciones de pensamiento de manera que puedan ayudar a que ellas y ellos den forma a sus propios saberes.

La preocupación por los saberes y las tradiciones de pensamiento que merece la pena poner a disposición de nuestros estudiantes, y por el tipo de mediaciones que somos capaces de favorecer, es una tarea necesaria como docentes (Blanco, 2006); y el modo en que damos forma a esa preocupación está íntimamente ligada a nuestras propias concepciones acerca de lo que entendemos que es un educador o una educadora.

\section{Sobre el sentido de ser educadora o educador}

\footnotetext{
${ }^{6}$ Nos estamos refiriendo a una predilección hacia talleres y dinámicas grupales, así como a las prácticas externas que realizan como parte de su formación, de modo que existe una tendencia bastante general de rechazo a las lecturas, o de dificultad para que las lecturas y el estudio (entendido como trabajo intelectual) se integren con verdadero sentido en su formación. En este sentido, un camino que se abre para explorar, tal y como hemos ido comprobando a lo largo del curso, es la recurrente tendencia a rehusar el trabajo eminente teórico, aun cuando tratamos de que esté ligado a la exploración e interpretación de la experiencia.
} 
Pensamos que ser una educadora, ser un educador, requiere algo más que saber qué hacer y cómo hacerlo. La educación es siempre relación, encarnada y viva (Blanco, 2006), y requiere que cada una, que cada uno, ajuste cuantas consigo mismo; por eso se dice que "educamos como somos" (Novara, 2003). De acuerdo con esto, entendemos que la formación requiere aprender no sólo - ni tanto - conocimientos, sino aprender a conocer-nos y a desarrollar saberes propios. La formación inicial debe ser una oportunidad para aprender a actuar en primera persona, como quien se es, partiendo de sí (Sofias, 2007); tomando conciencia de nuestro propio ser pedagógico (Van Manen, 1998) y asumiendo la responsabilidad sobre las propias acciones.

En la escuela y en otros espacios con intencionalidad educadora, no se realiza un trabajo en el sentido corriente, sino que estamos ante actividades primarias de la vida en sociedad, de la vida en relación que permiten una convivencia más ordenada, pacífica y rica (Muraro, 2010; Piussi, 2010); actividades que sostienen y refuerzan el tejido social, apoyando la re-creación de la cultura. En este sentido, la labor educativa juega un papel central en la vida personal y colectiva, ya que contribuye a tejer y sostener los vínculos que hacen posible la vida-en-común.

Este es un propósito que necesita ser pensando continuamente, que necesita de una apuesta viva y vivaz para no verse "liquidado" (Piussi, 2008), o escondido bajo el manto de los discursos fatalistas y apesadumbrados sobre la vida social - en general - y sobre la educación - en particular.

Dentro de nuestra asignatura tratamos de hacernos eco de las palabras de Anna Maria Piussi a la hora de pensar acerca de la labor que las educadoras y los educadores sociales llevan a cabo acompañando el malestar ${ }^{7}$ y el desorden de muchas mujeres y muchos hombres; poniéndose del lado de personas en situaciones vitales comprometidas. $Y$ tratamos de trabajar acerca del sentido propiamente educativo de esa actividad, que es algo distinto a proveer recursos o redirigir a usuarios y usuarias a distintos programas.

Es cierto que los cambios y las transformaciones sociales están afectando a las expectativas que la sociedad mantiene respecto de las instituciones educativas y formativas; como también afecta al propio sentido de lo que en ellas se trata de hacer (¿qué es hoy y hacia dónde va la educación?), y a las demandas que se generan sobre las y los profesionales de la educación.

Bajo este contexto de incertidumbre aparece la tendencia a tratar de definir - que quiere decir, dar un significado - lo que es o debiera ser una educadora o un educador; y ahí la formación inicial se configura alrededor del empeño por diseñar perfiles profesionales bajo catálogos de competencias. Sin embargo, ser una educadora, un educador, es algo más - y bastante más complejo - que tener un perfil profesional bien acotado. Estos perfiles dicen acerca de lo que se considera valioso que sepan hacer los profesionales. Pero lo que no dicen es cómo deben ser las educadoras y los educadores, no hablan acerca del sentido; y en educación, es el ser lo que define el sentido del hacer.

Y es esto precisamente lo que rara vez se aborda en la formación: quién se es, qué vivencias han resultado significativas en la constitución de nuestro ser pedagógico; y, a partir de este tipo de interrogantes, qué clase de mediaciones hemos de procurar desde la formación para que esto se movilice fruto del pensamiento, y pueda dar lugar a saberes profesionales que nos orienten en la acción.

Hay ahí un abismo de sentido muy difícil de salvar, entre lo que habitualmente proponemos como trabajo académico, y las experiencias vitales de las y los estudiantes. Y es ahí donde cobra valor organizar la Formación Inicial a partir de la experiencia.

\footnotetext{
${ }^{7}$ La noción de malestar que manejamos está apoyada en el trabajo de Cristina Brullet y Carme Gómez-Granell (2008). Según las autoras (pp. 11-12), el malestar tiene que ver con los estados de ánimo y sentimientos de desafiliación, desvinculación, inseguridad, aislamiento, presión desmesurada, angustia o frustración; sentimientos vinculados a las heridas que las fuerzas sociales pueden infligir en la experiencia humana.
} 


\section{El saber de la experiencia y la formación}

Cuando hacemos un corte con la experiencia y presentamos el conocimiento en forma de discurso - en el aire, sin origen -, se hace muy costoso hacer con él algo que tenga verdadero sentido. Dice Luigina Mortari (2002) al respecto que "al saber escrito de los libros le falta el aire, no permite espacios de respiro y no deja respirar a las mentes porque precisamente sufre de una desarraigo de la realidad, de la falta de la autenticidad de la experiencia" (p. 161). Y continúa: "Hay en el saber [refiriéndose al conocimiento académico] una cierta arrogancia que provoca la impresión de que dice todo lo necesario y de que ya no tiene sentido buscar más allá porque todo queda explicado en él." En un sentido parecido se pronuncia Max van Manen (2003) cuando señala que la literatura educativa nos puede proporcionar unos importantes conocimientos: "pero son unos conocimientos externos. No nos proporciona sin más un mayor tacto ni mayor sensibilidad en nuestras relaciones de todos los días con los jóvenes" (p. 15).

De nuevo el interrogante que como formadores se nos plantea tiene que ver con las mediaciones con el saber, teniendo presente que "prepararse para el oficio educativo requiere favorecer el saber de la experiencia: reconocerlo, re-elaborarlo, desarrollarlo, prepararse para contar con él. Requiere favorecer el desarrollo de un saber pedagógico personal que nace del preguntarse por el sentido de la experiencia vivida" (Contreras, 2011, p. 61).

Nos sentimos comprometidos en un tipo de indagación y de práctica que es sensible al valor del saber de la experiencia, y que considera que la Universidad puede y debe tener un papel clave en elaborar y transmitir un conocimiento pedagógico capaz de conectar con la experiencia viva de quienes enseñan/educan. Un saber que nace de lo vivido pero que requiere haber pasado por la reflexión, por el pensamiento. El saber de la experiencia es, dirá Luigina Mortari (2002), "el que mantiene una relación pensante con el acontecer de las cosas, el de quien no acepta un estar en el mundo según los criterios de significación dados sino que va en busca de su propia medida" (p. 155).

Por propia experiencia hemos ido aprendiendo que el conocimiento necesario en la formación de educadoras y educadores es aquel capaz de tener resonancia en la memoria, en singular y en contexto, de alguien que puede transformar lo vivido en saber, al pensar sobre lo que vive y lo que le sucede al vivirlo. Porque la experiencia no tiene tanto que ver con lo que pasa, con lo que hacemos, cuanto con lo que me pasa, con la resonancia que tiene en mí.

Transmitir el saber de la experiencia es complejo. No resulta sencillo disponer de relatos, de narraciones que respeten la naturaleza de ese saber; que mantengan esa cualidad de estar vinculado a quien lo ha producido, de permanecer cerca de la vida con lo que ello tiene también de irrepetible. María Zambrano dirá que el saber tiene su propio método de transmisión y que hay que aceptar que parte de él quedará siempre en secreto, porque no se puede o no se quiere comunicar. Pero su potencia está en otro lugar, en la verdad que representa y que la filósofa expresa así: "La experiencia irrenunciable se transmite únicamente al ser revivida, no aprendida. Y la verdad, la que la vida necesita, sólo es la que en ella renace y vive, la que es capaz de renacer tantas veces como sea necesitada" (Zambrano, 2000, p. 86).

Tampoco basta con disponer de esos relatos (vivos o a través de narraciones) sino que es necesario organizar situaciones pedagógicas que posibiliten la escucha de ese saber, y el diálogo con la propia experiencia.

En nuestras prácticas, tratamos de abordar esa complejidad de que hablamos, recuperando la matriz relacional (Blanco, 2006) del conocimiento, en un triple plano:

a) en el lugar de la creación del conocimiento, buscando el vínculo con el contexto

en el que fue creado y con las personas que lo mantienen vivo; 
b) en la transmisión, buscando crear relaciones de autoridad con las y los estudiantes, y también con el conocimiento - enseñando aquello que creemos que tiene

potencialidad educativa, aquello que ha pasado por nosotros;

c) en la relación de cada estudiante con el conocimiento profesional, buscando el

vínculo con su propia experiencia.

Esto se traduce en pensar en unas formas de enseñanza que buscan restablecer el vínculo de los saberes con quienes los han desarrollado; que priorice el conocimiento de una misma, de uno mismo; que nos posibilite conocer nuestras ideas, reflexionar sobre ellas; que nos permita el diálogo con sí mismo y con las y los demás; que nos enseñe a convivir con la incertidumbre orientándonos por el sentido de lo que hacemos y no sólo, ni tanto, por los resultados de nuestras acciones.

\section{Formar-se y conocer-se: aprender a escuchar-se}

La formación, tanto inicial como permanente, debe constituir una oportunidad para desarrollar los propios modos de actuar, para madurar y modular el alcance de nuestros deseos y conocer las concepciones que sostienen nuestras prácticas. No es sólo disponer de recursos para actuar; es desarrollar una disposición hacia la reflexión, el análisis de la propia experiencia y la escucha atenta hacia las otras y los otros. Formar es formar-se, desarrollar la sensibilidad pedagógica que nos permite responsabilizarnos de nuestras acciones, orientadas siempre al bienestar de aquellas personas con las que nos relacionamos.

Los planteamientos de Daniele Novara (2003) tienen que ver con entender la formación como una experiencia en la que podamos tomar conciencia de uno mismo, de una misma como educador. Para este autor, esta iniciación al autoconocimiento es crucial para educadoras y educadores. Pero, ¿qué entendemos por autoconocimiento y qué relaciones hay entre este proceso de mirar-se y la formación? Para Novara, el autoconocimiento tiene que ver con hacernos más conscientes de los móviles que fundamentan y dan sentido a nuestro proyecto educativo; y nuestro proyecto educativo alude tanto las ideas que proclamamos como a aquellas que desplegamos en la acción, y que a menudo se parapetan tras los discursos declarativos. De eso se trata entonces, de abrir la posibilidad para dialogar, personal y colectivamente, entre ambos planos, ya que conformar saberes profesionales necesita que pongamos en juego no sólo nuestro discurso formal, sino nuestra historia de vida (Correa, 1999).

Lo que propone Novara es una visión cultural del inconsciente pedagógico, para lo que plantea la necesidad de abordar preguntas tales como: ¿De quiénes hemos aprendido a hacer de educadores? ¿Qué y cómo permanece en nosotros aquello que nuestros padres y madres "hicieron con nosotros" (nuestra crianza)? ¿Qué permanece en nosotros, en nosotras, del paso por nuestras vidas de otras educadoras y otros educadores? ¿Cómo influye todo esto en nuestras subjetividad, en nuestros marcos de interpretación de los acontecimientos en que nos vemos inmersos, y en nuestro estilo de relación pedagógica? Y como pregunta de fondo, ¿̇en qué grado somos conscientes de esto y hacia dónde movilizarlo?

En nuestro trabajo, nos apoyamos parcialmente en la propuesta de este autor: explorar los escenarios educativos vividos (por lo general, nuestra biografía como estudiantes) y nuestras vivencias de relación pedagógica (en general, como hijas e hijos), para ayudarnos a pensar cómo nuestras vivencias como sujetos educados condicionan fuertemente tanto nuestros estilos cognitivos como nuestras modalidades de aprendizaje. Es fundamentalmente en esa clase de vivencias donde hemos ido conformando el sentido acerca de lo pedagógico, tanto en un plano declarativo (cuál es nuestro discurso acerca de lo deseable en educación) como en un plano relacional (cómo nos ponemos en juego, cómo habitamos los escenarios educativos). Esto es algo que aparece recurrentemente en los escritos de las alumnas y de los alumnos cuando indagan sobre el origen de su deseo de ser educadoras y educadores. 
Como propuesta formativa, la exploración reflexiva de la experiencia trata de ser una mediación entre nuestras ideas, convicciones, posiciones morales y emocionales, y nuestra acciones (o propuestas de acción). Hay distintas orientaciones sobre la virtualidad pedagógica de la exploración biográfica (Novara, 2003; Rivas y Herrera, 2009), en nuestro caso, el relato biográfico adquiere sentido como un modo de dialogar con nuestra historia de vida, fundamentalmente en aquellas vivencias que permitan situar la reflexión sobre lo educativo, y que favorezca tanto reconocer los nudos emocionales que no hemos resuelto (y que podrán verse reactivados en las relaciones pedagógicas), como conectar con las personas y los saberes que sostienen nuestro ser pedagógico ${ }^{8}$.

Poner pensamiento a las vivencias es una de las tareas de la formación que puede sacudir los cimientos de lo que a menudo creemos que son unos esquemas asentados y coherentes. Abordar esto nudos es central en nuestra tarea de enseñar, de formar a educadoras y educadores. Porque cuando vamos identificándolos y los explicitamos en clase, cuando hacemos algo con ellos, estamos abordando asuntos nucleares de su ser educadores. Como puede entenderse, estamos ante un asunto de una extraordinaria delicadeza, en la que los sujetos se exponen, mostrando algo sustancial de su intimidad. De ahí que esta sea una opción formativa que requiere de un especial cuidado de las relaciones en el aula, de los vínculos que seamos capaces de favorecer. Unas relaciones que buscan orientarse desde la autoridad y no - o lo menos posible - desde el poder (Piussi, 2001; Blanco, 2002).

Así pues, crear y sostener relaciones de autoridad ${ }^{9}$ constituye el sustrato que alimenta las opciones metodologías y de selección de materiales y actividades que puedan concretar la propuesta de formación. Opciones que incluyen:

a) seleccionar, como fuente de saber profesional, narraciones de educadoras y educadores que reflexionan sobre su práctica;

b) privilegiar la presencia viva de educadores y educadores en la Universidad, como parte de las actividades de formación;

c) demandar el relato de experiencias propias de las alumnas y los alumnos;

d) utilizar las experiencias vividas por las y los estudiantes como fuente de aprendizaje;

e) favorecer los procedimientos de valoración que toman como base el desarrollo de saberes propios: la elaboración de ideas frente a la repetición de conocimientos establecidos.

\footnotetext{
${ }^{8}$ El campo de la investigación biográfica y narrativa posee un importante recorrido intelectual y práctico a lo largo de los últimos 30 años. Dentro de este campo de indagación, nuestras posiciones guardan relación con lo que Gil Cantero y Jover (2000) llaman las tendencias narrativas en pedagogía. Según los autores podemos incluir bajo esa clasificación "desde estudios teóricos encaminados a conceptualizar a la educación como un acontecimiento narrable, hasta propuestas prácticas sobre los efectos formativos de animar a los educandos, y a los educadores, a que narren y valoren sus propias vidas" (p. 111).

${ }^{9} \mathrm{La}$ idea de autoridad a la que nos referimos es la que sostienen las mujeres del pensamiento de la diferencia sexual, autoridad nombrada como autoridad femenina (Rivera, 2005). En este sentido, la autoridad no se da, no se impone, sino que es entendida como una concesión que alguien hace sobre otra persona, basada en la confianza que le tiene, y que le hace fiarse de ella.
} 


\section{Una propuesta de trabajo para favorecer la emergencia del saber de la experiência}

Nuestra propuesta de trabajo se articula en torno a una variedad de actividades entre las que se priorizan: la lectura y análisis de relatos biográficos y de relatos de educadoras y educadores sociales; análisis de experiencias que reflejan valores educativos sobre los que vale la pena pensar; o narrar el sentido de la elección de ser educadora o educador. Estas tareas las abordamos favoreciendo la escritura y el diálogo partiendo de sí (qué significan para mí, no en abstracto); solicitando la justificación de las elecciones que hacemos (no es tan importante la elección en sí como las razones por las que elegimos); y elaborando el propio proceso de aprendizaje en un proceso que implica enfrentarse a la inseguridad y afrontar la incertidumbre.

Las actividades propuestas se articulan en espacios de formación (Contreras, 2011, p. 62) que buscan ser lugares para la exploración y el desarrollo del saber de la experiencia: a) la experiencia vivida; b) la experiencia de educadoras y educadores; c) las clases como lugar de experiencia.

\section{+ La experiencia vivida ${ }^{10}$}

A través de diversas actividades, como el relato de su elección de ser educadoras y educadores, y en todas las ocasiones que nos es posible, buscamos la conexión con lo que han vivido para pensarlo, para ser conscientes del modo en que influye en sus decisiones, para que puedan reconocer los vínculos entre distintos contextos de acción, para que puedan reconocer de dónde proceden las cualidades que valoran en su profesión (y en la vida) y que han aprendido - con frecuencia, como nos han contado - en su casa, con su madre.... Así lo reflejan en sus relatos, donde las madres son - sobre todo en las chicas - un referente muy presente, aunque a menudo no han pensado en ello ni en el sentido que tiene.

Y hemos encontrado de gran valor el trabajo con relatos biográficos (a menudo publicados como relatos literarios), como un modo de acercarles a la reflexión sobre sus propias concepciones, enfrentarles a sus prejuicios, sus miedos; a poner en cuestión sus creencias, a pensar en sí a partir del reflejo de otras y otros. $\mathrm{Y}$, entre otros, nos enfrentamos al reto de enseñar a pensar y a escribir partiendo de sí. ¿Cómo se favorece esa disposición? ¿Hasta dónde podemos pedirle que se expongan, que indaguen en sus concepciones, que muestren sus contradicciones? Se juega aquí la diferencia entre intimidad y privacidad, entre subjetivismo y subjetividad que no pueden resolverse de una vez por todas ni de una manera discursiva. Hay que hacerlo en cada caso, en el contexto del aula, en un clima de confianza y apertura que no está dado, sino que hay que ir creando y cuidando.

\section{+ La experiencia de educadoras y educadores}

Tanto a través de narraciones escritas, como de visitas, buscamos que los saberes de educadoras y educadores constituyan un referente fundamental en su formación porque, como hemos indicado, es un saber encarnado, contextualizado, complejo y capaz de desbordar los límites del conocimiento disciplinar para estar más cerca de la realidad. Y no es tarea sencilla ni automática encontrar relatos que tengan unas determinadas cualidades; relatos que den cuenta del saber de la experiencia, que no sólo cuenten - en el sentido de describir y narrar - sino que incorporen una

\footnotetext{
${ }^{10}$ Desde la fenomenología hermenéutica, van Manen (2003) aclara que hay una clara diferencia entre lo vivido, y lo vivido sujeto a la reflexión. Es a partir de esa reflexión sobre el acontecer de las cosas, a lo que en el artículo nos hemos referido como "poner pensamiento a lo vivido ". En un sentido parecido, esta vez desde la etnosociología, Bertaux (2005) diferencia entre existencia y experiencia, poniendo el acento en cómo la reflexión sobre las vivencias otorgan un nuevo grado de saber sobre la experiencia.
} 
reflexión sobre lo vivido, que dejen ver el sentido de lo que se hace y no sólo de las acciones; que parten de sí y no son meros relatos de acción.

Relatos que muestren cualidades relevantes de la práctica o del conocimiento de educadores o educadores, que muestren principios de acción y orienten el sentido de la misma. Que posibiliten el diálogo y la reflexión de quienes los leen, que muestran un modo de pensar y de pensar-se, un saber que toma cuerpo en alguien concreto y singular. Relatos que ofrecen "recetas" en el sentido que - para ese término - rescata Ana Mañeru (2002): alguien que narra lo que hace para ponerlo a disposición de otras y de otros, pero que no puede copiarse ni reproducirse al modo en que lo hacen las fórmulas, y que también se aleja de la abstracción.

Buscamos que la experiencia de otros nos abra a preguntas relevantes, que nos permitan pensar y - sobre todo - pensarnos. Que nos interpelen, que hablen a nuestra experiencia, que hagan viable y necesario el diálogo con nuestras ideas, que nos interroguen sobre nuestras concepciones, que nos sostengan en las dudas y en la búsqueda de sentido propio.

\section{+ Las clases como lugar de experiencia}

Encontrar textos valiosos es importante, como lo es posibilitar centrarse en la experiencia vivida. Parte importante de la potencialidad que tengan está en encontrar el modo de que las clases sean un lugar de experiencia. Un lugar donde haya oportunidades para ponerse en juego, para dialogar con la experiencia de otras y de otros, para vivir situaciones a partir de las que pensar y pensar-se; para ofrecer-se y, también, para dejar-se dar. En el aula hay que crear un contexto que permita que esos saberes de la experiencia nos afecten, que nos dejemos tocar por ellos, que sintamos con ellos, experimentando que lo que sabemos y lo que somos está imbricado, que pasa por nosotras y nosotros, que nos transforma. Y para ello hemos de consentir, hemos de dejarnos transformar, de dejarnos tocar.

Encontramos que es un reto importante disponer un ambiente para preparar al alumnado en un tipo de reflexión que no es una estrategia sino una disposición, una actitud, algo que no puede convertirse en una rutina. Algo que requiere contenidos relevantes y un clima de confianza en nosotros como docentes. Un clima y unos contenidos que posibiliten partir de sí como colocación específica frente a nosotros mismos y en relación a la realidad, asumiendo la responsabilidad de nuestro vínculo. Un vínculo que siempre es en primera persona (nunca abstracto), nos exige apoyarnos en nuestra experiencia, en quienes somos, y definir nuestra relación con la realidad que vivimos; partir de sí es un modo de implicarnos en esa realidad, asumir la responsabilidad de hacer lo que está en nuestra mano para que sea más civilizada, por amor al mundo (como pedía Hannah Arendt).

Y un reto también en enseñar que hablar desde sí no es hablar de sí, no es contar tu vida sino pensar sobre lo vivido desde ti, evitando la abstracción y la generalización, el relato subjetivista, el contar anecdótico e irreflexivo. Favorecer la indagación sobre el sentido de lo que viven, sabiendo que la búsqueda de diálogo profundo y auténtico consigo requiere una relación de confianza, de autoridad. Algo enormemente difícil en los contextos escolares, tan cargados y tan delimitados por las calificaciones, por el intercambio de actuaciones por calificaciones. Y no es menor, pensamos, la dificultad que tenemos para valorar sus experiencias como estudiantes porque descubrimos que, a menudo, nos colocan en una posición de desconfianza hacia lo que puedan decir con sentido de autoría. Y para situar apropiadamente la propia dificultad que tienen, sobre todo los chicos, para pensar desde sí y entablar una comunicación en primera persona.

Por eso, además de explicitar los pilares en los que tratamos de asentar nuestra práctica, es importante que el aula sea también un lugar para mostrar - como docentes - nuestro propio saber de experiencia, la relación que tenemos con nuestro hacer, las reflexiones que nos provocan, los miedos e incertidumbres que tenemos, las seguridades provisionales, nuestros deseos y nuestras 
frustraciones... Mostrarse como ejemplo vivo de lo que les pedimos que hagan, sin impostar un papel.

\section{Principios que orientan - y que concretan - nuestras prácticas}

Los principios de procedimiento que orientan nuestras decisiones, y que buscamos que se materialicen en nuestras acciones, se centran en: sostener relaciones de autoridad en el aula, atender la singularidad de cada estudiante, practicar la escucha activa y promover el diálogo reflexivo.

\section{Sostener relaciones de autoridad en el aula}

Tratamos de crear y sostener una clima de relaciones que sea apacible, donde poder conocernos, expresarnos, y aprender desde la confianza (y lo menos posible desde el temor o la indiferencia). Vivir en el aula que la relación pedagógica siempre es de a dos, reconocimiento la singularidad de cada alumna y de cada alumno, que son únicos en lo que aportan a la clase, al mundo. La relación personal, nombrarles, contar con ellos, interesarnos cuando faltan etc., supone un telón de fondo donde ir engarzando el trabajo sobre la experiencia porque les identifica como quienes son: con un nombre, una historia, unas circunstancias.

Su presencia en la clase para nosotros es importante y es singular; cada proceso de aprendizaje es de alguien, y esa atención a lo singular entendemos que es una plataforma para que cada una y cada uno vaya haciendo su recorrido "no el aire" sino ligado a su propia biografía.

\section{Atender la singularidad de cada estudiante}

Nos esforzamos por escuchar la vida del aula: las miradas, los cuerpos, las palabras, los silencios, las presencias y las ausencias. Escuchar para orientar, dirigir, encauzar, llevándoles a recorrer un camino con unas características específicas, que lo ajena de lo general, de lo abstracto e insistiendo en ir a lo que me pasa, lo que a mí me dice.

\section{Practicar la escucha activa y promover el diálogo reflexivo}

Buscamos favorecer el diálogo, la escucha de sí y de las otras y otros. A veces esto requiere insistir en la necesidad de argumentar, de mirar con más detalle, de reconsiderar una idea (evitando que lo vivan como un reproche o una descalificación), buscando comprender en lugar de confrontar o polemizar; buscando que nuestra solicitud de esfuerzo por hacerse entender pueda ser una práctica de dar medida, esto es, tratando de encarnar la evaluación en la relación (Armellini, 2010).

Como soporte del trabajo cotidiano, que pueda reforzar la confianza y seguridad de las alumnas y de los alumnos, nos esforzamos por ofrecerles una planificación ordenada, explícita, que consultamos con ellas y ellos y a la que volvemos para reorientar el trabajo cuando es necesario. Y cuidamos que tengan los apoyos necesarios - en forma de guías - para enfrentar las tareas propuestas.

Y un aspecto que vivimos como sustancial es la posibilidad de realizar la tarea docente no en solitario, sino "de a dos"; una oportunidad poco común, como es trabajar al tiempo en el aula dos personas, compartiendo el diseño, el desarrollo y la valoración de las sesiones de trabajo. Ser una mujer y ser un hombre, tener más experiencia docente y ser más joven, se constituyen en una fuente de riqueza que buscamos traducir en posibilidades para nosotros mismos y también para el alumnado.

\section{La perspectiva de las alumnas y de los alunos}

No es la primera vez que organizamos la enseñanza buscando que la experiencia esté en el centro de la misma; aunque sí hemos indagado sobre su potencialidad y sus dificultades de un modo 
explícito, a través de un proceso de auto-estudio (Cochran-Smith y Lytle, 2002), como antes se expuso. Un descubrimiento importante ha sido que, para acercarnos al significado que tiene para las alumnas y los alumnos trabajar tomando como eje de sentido la experiencia - la propia y la de otras y otros -, no es apropiado preguntarles directamente. Es más conveniente analizar cómo se refleja en su reflexión sobre el propio proceso de aprendizaje. Cuando les preguntamos directamente qué sentido le daban y qué les había supuesto pensar sobre su propia experiencia o poder dialogar con la de otras y otros, la mayoría no supo qué responder; y quienes lo hicieron - sin duda llevados por su deseo de atender nuestra demanda - nos dieron respuestas que parecían forzadas y que no alcanzaban a tocar lo que considerábamos que podría ser valioso.

En consecuencia, nos preguntamos si, en realidad, había tenido alguna incidencia en su proceso de formación. O, quizá, es que no habíamos buscado del modo apropiado; porque acercarse a pensar la experiencia no es a través de la racionalización (en cierto modo, como decíamos con Zambrano (2002), se trata de un saber que en parte queda oculto), de la traducción en un relato ordenado y explícito. Más bien se traduce en una disposición a pensar desde sí, en ser parte del relato que se construye. En última instancia no se traduce tanto en un contenido específico como en una colocación simbólica, en un modo de estar presente en lo que se dice y se escribe, dando un sentido propio al pensar y al decir.

Leyendo la tarea final del semestre que les pedimos que realizaran - un relato reflexivo sobre su proceso de aprendizaje - hemos podido constatar el valor que ha tenido el modo en que hemos estado trabajando, y que se muestra en su capacidad para pensar desde sí, para buscar dar sentido a lo que han conocido y vivido. Y no es baladí que, para muchas y muchos, las prácticas que han realizado en el periodo inmediato a la escritura de esta tarea, haya constituido un referente increíblemente potente para articular pensamiento y sentimiento. Es de esos relatos de donde hemos extraído algunas ideas que han explicitado en relación con el valor de poner la experiencia en el centro del aprendizaje. Ideas que sintetizamos a continuación ${ }^{11}$.

Isabel reconoce la potencialidad de trabajar con textos que ayudan a construir el sentido de lo educativo "en cada uno de nosotros"; y valora como una ayuda "poder contar con experiencias que explican de forma clara y sencilla el sentido verdadero de la práctica de la educación social". Textos que animan y orientan respecto a la práctica, que les permiten pensar sobre el sentido de su elección de ser educadoras y educadores:

Estos textos, de alguna forma, animan a todas aquellas personas que se puedan sentir perdidas a la hora de enfrentarse a la realidad. Personalmente me han hecho

cuestionar las razones por las que he elegido esta profesión y a plantearme si son sólidas (Clara).

Textos que les ayudan a descubrir sus propias ideas, a tener criterio profesional, a conectar con sus experiencias:

Ha contribuido a construir mi propia identidad personal y profesional, pues hemos dedicado tiempo a reflexionar acerca de lo que queremos ser, en qué posición nos queremos colocar [...] (Marisa).

Por ello, tienen consciencia de que les enfrenta a sí mismas. Así lo expresa Reme, que - tras reconocerse como una persona "muy cuadriculada", que necesita tenerlo todo controlado - se confronta con la lectura de la experiencia de una educadora y comienza a moverse, y a desplazar su preocupación por querer cambiar el mundo "cambiar la relación con el mundo". Y ello porque, como algunas explicitan, han tenido la posibilidad de conocerse mejor, de "aprender a expresar los sentimientos" (Laia).

\footnotetext{
${ }^{11}$ Los nombres que aquí utilizamos son seudónimos; las estudiantes han autorizado la publicación de sus
} valoraciones. 
Coral dice haber descubierto que no era la timidez lo que le impedía intervenir en clase, sino el miedo a equivocarse.

Este encuentro conmigo misma, con mis emociones más ocultas, me han surgido mediante la lectura de unos textos, las experiencias de algunos autores con los que he descubierto que ser mejor profesional no está ligado -siempre y en todo- con los estudios, sino también con encontrar tu lugar, dejando sitio a las emociones y también a nuestros errores para así poder aprender de ellos y confiar más en las propias possibilidades (Coral).

$\mathrm{Y}$ alguna reconoce el valor de dejar constancia de la propia experiencia, de la escritura como un modo tanto de mejorar profesionalmente como de apoyar a otras y a otros en su aprendizaje (Amelia). Trabajar con la propia experiencia ayuda a profundizar en la comprensión de lo que sabemos y sentimos, aunque a veces dé miedo. Escribir sobre lo que se ha aprendido es un desafío porque significa hablar de sentimientos y emociones (lo que te pasa);

Califico esto de desafío porque considero que hablar de sentimientos significa

desnudarse ante los demás (y a lo largo de mi vida siempre lo he evitado) (Nadia).

Por tanto, escribir sobre la propia experiencia, sobre lo que pensamos y sentimos, es un ejercicio necesario pero difícil:

Cuesta dejarse ver, me cuesta decir lo que realmente siento y no por el miedo a

equivocarme o a decir algo que "no encaje"; es el desnudarme, el dejar ver a otra

persona lo que significa para mí esta profesión (Lara).

Pero algo que valoran, porque más allá del miedo o el pudor, "hemos sacado cosas que no sabíamos que existían y nos han servido para dar sentido al presente" (Nadia).

Así pues, podemos decir que para las alumnas y los alumnos, haber participado en un proceso de formación que toma como eje de sentido la experiencia ha sido valioso en tanto les ha ayudado a ir construyendo el sentido de lo educativo en cada una y cada uno, a ir descubriendo sus propias ideas y a conocer los principios que orientan sus acciones. Tomar la experiencia propia como referente para el aprendizaje facilita profundizar en la comprensión de lo que sienten y piensan, pero también les enfrenta los miedos y los riesgos que se derivan de este exponer-se, ante sí y ante los demás.

\section{Retos y possibilidades}

Quizá precisamente porque estamos teniendo la posibilidad de continuar durante un curso completo con el mismo grupo de estudiantes, tenemos la fortuna de poder ir descubriendo tanto las posibilidades como los retos (las dificultades) que enfrentamos al organizar la enseñanza del modo en que venimos contando. Algunas de esas dificultades pueden tener que ver con nuestras propias limitaciones como docentes, con la necesidad de analizar con más detenimiento (de seguir analizando quizá sería mejor decir) las inconsistencias o debilidades que hay en el modo en que materializamos nuestros propósitos. Pero otras tienen muy claramente una dimensión institucional y requieren otro tipo de abordaje.

Decía María Zambrano (2000) que el maestro ha de llegar para dar tiempo y luz. El tiempo es imprescindible para las relaciones, para facilitar el conocimiento de sí y en relación a la profesora o el profesor, para madurar ideas, para el trabajo sosegado y para que pueda fructificar ese trabajo más allá de una asignatura, como soporte para entender-se y conocer mejor su campo de actuación. En nuestro caso, las prácticas que han realizado inmediatamente después de finalizar la asignatura y simultáneas a la tarea de reflexión para elaborar el escrito con que cerrarla, ha constituido un referente increíblemente potente para articular pensamiento, sentimiento, reflexión y emoción. Todo ese aprendizaje está constituyendo, además, una potente base sobre la que estamos asentando el 
trabajo y las relaciones en el segundo semestre. La compresión del tiempo que estamos viviendo en la Universidad, generalizando el semestre como la unidad temporal para la docencia, no favorece establecer relaciones pedagógicas de confianza y sosiego, imprescindibles para la formación.

Otro reto fundamental que abordar es el de la evaluación. Más allá de buscar formas y procedimientos coherentes con los principios de la enseñanza, el contexto de calificación y competitividad de la formación universitaria constituye un importante obstáculo. La estructura de relaciones de poder que subyace a las prácticas de calificación pueden bloquear el establecimiento de las relaciones de confianza y autoridad. Es una tarea delicadísima y, como hemos podido comprobar en la experiencia que estamos analizando, genera conflictos y malentendidos que requieren ser trabajados con un exquisito cuidado. La evaluación es una práctica que para que tenga sentido, debe sostenerse dentro de una relación de autoridad. Cuando un estudiante confía en el juicio de su profesora, de su profesor, está en disposición de acoger la valoración de un trabajo, de una tarea; y sólo así podemos hablar de evaluación educativa. De ahí la importancia que le otorgamos a las relaciones pedagógica: entablar un vínculo, tratar de erigirnos como referentes de quienes se fíen, es un principio de acción vertebrador de nuestra práctica. Y para ello, también es fundamental disponer de tiempo para cuidar las relaciones.

\section{Referencias}

Arendt, H. (2004). La condición bumana. Barcelona: Paidós.

Armellini, G. (2010). Miseria y nobleza de la evaluación. En Lelario, A.; Cosentino, V.; Armellini, G. Buenas noticias de la escuela. Hechos y palabras del movimiento de autorreforma. Madrid: Sabina Editorial. p. 146-159.

Bárcena, F. y Mèlich, J.-C. (2000). La educación como acontecimiento ético. Barcelona: Paidós.

Blanco, N. (2002). Tratando de crear y sostener relaciones de autoridade en la educación. En Sofías, Escuela y educación. ¿Hacia donde va la libertad femenina? Edición al cuidado de $\mathrm{M}^{\mathrm{a}}$ Milagros Montoya. Madrid: horas y HORAS, p. 113-124.

Blanco, N. (2006). Saber para vivir. En Piussi, A.M. y Mañeru, A. (coords.). Educación, nombre común femenino. Barcelona: Octaedro. p. 158-183.

Blanco, N. (2010). La investigación en el ámbito del curriculum y como método para su desarrollo. En Gimeno, J. Saberes e incertidumbres sobre el curriculum. Madrid: Morata, p. 569-587.

Brullet, C. y Gómez-Granell, C. (Coord.) (2008). Malestares. Infancia, adolescencia y familias. Barcelona: CIIMU - Graó.

Cochran-Smith, M. y Lytle, S. L. (2002). Dentro/Fuera. Enseñantes que investigan. Madrid: Akal.

Contreras Domingo, J. (1991). El sentido educativo de la investigación. Cuadernos de pedagogía, no 196, p. 61-67.

Contreras Domingo, J. (2010). Ser y saber en la formación didáctica del profesorado: una visión personal. Revista Interuniversitaria de Formación del Profesorado, 68 (24,2), p. 37-60.

Contreras Domingo, J. (2011). El lugar de la experiencia. Cuadernos de Pedagogía, 417, p. 60-63.

Contreras Domingo, J. y Pérez de Lara, N. (comps.) (2010). Investigar la experiencia educativa. Madrid: Morata.

Correa, R. (1999). La aproximación biográfica como una opción epistemológica, ética y metodológica. Proposiciones 29, Marzo. Disponible en:

http://www.sitiosur.cl/publicaciones/Revista_Proposiciones/PROP-29/12CORREA.zip

Última descarga: 04-05-2012.

Cantero, F.G. y Jover, G. (2000). Las tendencias narrativas en pedagogía y la aproximación biográfica al mundo infantil. En Enrahonar 31, 2000: 107-122. Disponible en: 
http://www.raco.cat/index.php/enrahonar/article/viewFile/31982/31816

Última descarga: 03-06-2012.

Korthagen, F. (2011). Principios para una formación eficaz. Cuadernos de pedagogía, 417. p. 56-59.

López Carretero, A. (2006). Hacerse mediación viva. En Piussi, A.M. y Mañeru, A. (coords.), Educación, nombre común femenino. Barcelona: Octaedro. p. 134-157.

Loughran, J. (2004). International Handbook of Self-study of Teaching and Teacher Education Practices. London: Springer.

Mañeru, A. (2002). Escuela y educación. ¿Hacia donde va la libertad femenina? Edición al cuidado de $\mathrm{M}^{\mathrm{a}}$ Milagros Montoya. Madrid: horas y HORAS.

Mecenero, C. (2009). Passaggi: maestre tra scuola e universita'. L'esperienza delle supervisore dei corsi di laurea in Scienze Della formazione primaria: due Studio di caso. Tesis de Doctorado inédita. Dipartimento di Scienze dell'Educazione. Universita' degli Studi di Verona.

Mortari, L. (2002). Tras las huellas de un saber. En Diotima. El perfume de la maestra. Barcelona: Icaria. p. 153-162.

Muraro, L. (2010). Una excelencia contagiosa y amable. En Lelario, A.; Cosentino, V.; Armellini, G. Buenas noticias de la escuela. Hechos y palabras del movimiento de autorreforma. Madrid: Sabina Editorial. p. 27-32.

Novara, D. (2003). Pedagogía del saber escuchar. Madrid: Narcea.

Piussi, A.M. (2001). Dar classe: el corte de la diferencia sexual. En Blanco, N. (Coord.). Educar en feminino y en masculino. Madrid: Akal, p. 145-165.

Piussi, A. M. (2008). Posibilidad de una escuela de libertad. En Boff, L. et alt., Figuras y pasajes de la complejidad en la educación. Experiencias de resistencia, creación y potencia. Instituto Paulo Freire de España, Valencia.

Piussi, A.M. (2010). Un pensamiento político del educar. En Lelario, A.; Cosentino, V.; Armellini, G. Buenas noticias de la escuela. Hechos y palabras del movimiento de autorreforma. Madrid: Sabina Editorial. p.42-52.

Rivas Flores, J. I. y Herrera Pastor, D. (Coord) (2009). Vozy educación. La narrativa como enfoque de interpretación de la realidad. Barcelona: Octaedro.

Rivera Garretas, M. (2005). La diferencia sexual en la historia. Valencia: Publicaciones Universidad de Valencia.

Sáez Carreras, J. y Molina, J. (2006). Pedagogía Social. Pensar la Educación Social como profesión. Madrid: Alianza Editorial.

Santos Guerra, M. A. (2002). Una flecha en la diana. La evaluación como aprendizaje. En Revista Andalucía Educativa, no 34, 7-9.

Sofias (2007). Saber es un placer. La práctica política de mujeres que buscan dar sentido libre a la educación.

Edición al cuidado de $\mathrm{M}^{\mathrm{a}}$ Milagros Montoya. Madrid: horas y HORAS.

Tardiff, M. (2004). Los saberes del docente y su desarrollo profesional. Madrid: Narcea.

Van Manen, M. (1998). El tacto en la enseñanza. Barcelona: Paidós.

Van Manen, M. (2003). Investigación educativa y experiencia vivida. Barcelona: Idea-Books.

Zambrano, M. (2000). Hacia un saber sobre el alma. Madrid: Alianza. 


\section{Sobre os Autores}

\section{Nieves Blanco García}

Universidad de Málaga (España)

Licenciada en Pedagogía y Doctora en Ciencias de la Educación. Profesora en la Facultad de Ciencias de la Educación (Departamento de Didáctica y Organización Escolar) y coordinadora del Doctorado en Didáctica y Organización Educativa de la Universidad de Málaga. Miembro del comité editorial de diversas publicaciones especializadas. Autora de un centenar de contribuciones científicas en libros y artículos de revistas.

nblanco@uma.es

\section{José Eduardo Sierra Nieto}

Departamento de Didáctica y Organización Escolar, Facultad de Ciencias de la Educación, Universidad de Málaga (España).

Ldo. en Pedagogía por la Universidad de Málaga, Universidad en la que ha cursado Estudios de Doctorado en el Programa "La política educativa en la sociedad neoliberal". Educador social con menores en protección en la ONG PRODIVERSA (antes Movimiento por la Paz-Málaga), donde también coordinó tareas de formación. Pertenece al Grupo de Investigación "Evaluación e Innovación educativa andaluza", dirigido por el profesor Ángel I. Pérez Gómez, de la Universidad de Málaga. Investigador en formación en el Departamento de DOE, donde compagina la realización de su Tesis Doctoral sobre narrativas de fracaso escolar y masculinidades, dirigida por la profesora Nieves Blanco, con tareas de docencia relacionadas con el Diseño de Programas.

esierra@uma.es

\section{Sobre as Editoras Convidadas}

\section{Carla Beatriz Meinerz}

Universidade Federal do Rio Grande do Sul

Doutora em Educação pela Universidade Federal do Rio Grande do Sul. Professora Adjunta no Departamento de Ensino e Currículo da Faculdade de Educação.

carlameinerz@gmail.com

\section{Dóris Maria Luzzardi Fiss}

Universidade Federal do Rio Grande do Sul

Doutora em Educação pela Universidade Federal do Rio Grande do Sul. Professora Adjunta no Departamento de Ensino e Currículo da Faculdade de Educação.

fiss.doris@gmail.com

\section{Sônia Mara Moreira Ogiba}

Universidade Federal do Rio Grande do Sul

Mestre em Educação pela Universidade Federal do Rio Grande do Sul. Professora do

Departamento de Ensino e Currículo da Faculdade de Educação. Membro da Associação

Psicanalítica de Porto Alegre (APPOA) e Instituto APPOA - Clínica, Pesquisa e Intervenção

Social.

ogb@cpovo.net 


\section{DOSSIÊ \\ FORMAÇÃO DE PROFESSORES E PRÁTICAS CULTURAIS arquivos analíticos de políticas educativas

\section{(c)}

SORERIGHIS RESERED O Copyright e retido pelo/a o autor/a (ou primeiro co-autor) que outorga o direito da primeira publicação à revista Arquivos Analíticos de Políticas Educativas. Más informação da licença de Creative Commons encontram-se em http://creativecommons.org/licenses/by-nc-nd/2.5. Qualquer outro uso deve ser aprovado em conjunto pelo/s autor/es e por AAPE/EPAA. AAPE/EPAA é publicada por Mary Lou Fulton Institute Teachers College da Arizona State University. Os textos publicados em AAPE são indexados por CIRC (Clasificación Integrada de Revistas Científicas, Espanha) DIALNET (Espanha),Directory of Open Access Journals, Education Full Text (H.W. Wilson), EBSCO Education Research Complete, , ERIC, , QUALIS A2 (Brasil), SCImago Journal Rank; SCOPUS, SOCOLAR (China). Contribua com comentários e sugestões a http://epaa.info/wordpress/ ou para Gustavo E. Fischman fischman@asu.edu.

Curta a nossa comunidade EPAA's Facebook https://www.facebook.com/EPAAAAPE e Twitter feed@epaa_aape. 


\section{arquivos analíticos de políticas educativas conselho editorial}

Editor: Gustavo E. Fischman (Arizona State University) Editores Associados: Rosa Maria Bueno Fisher e Luis A. Gandin

(Universidade Federal do Rio Grande do Sul)

Dalila Andrade de Oliveira Universidade Federal de Minas Gerais, Brasil

Paulo Carrano Universidade Federal Fluminense, Brasil

Alicia Maria Catalano de Bonamino Pontificia Universidade Católica-Rio, Brasil

Fabiana de Amorim Marcello Universidade Luterana do Brasil, Canoas, Brasil

Alexandre Fernandez Vaz Universidade Federal de Santa Catarina, Brasil

Gaudêncio Frigotto Universidade do Estado do Rio de Janeiro, Brasil

Alfredo M Gomes Universidade Federal de Pernambuco, Brasil

Petronilha Beatriz Gonçalves e Silva Universidade Federal de São Carlos, Brasil

Nadja Herman Pontificia Universidade Católica -Rio Grande do Sul, Brasil

José Machado Pais Instituto de Ciências Sociais da Universidade de Lisboa, Portugal

Wenceslao Machado de Oliveira Jr. Universidade Estadual de Campinas, Brasil
Jefferson Mainardes Universidade Estadual de Ponta Grossa, Brasil

Luciano Mendes de Faria Filho Universidade Federal de Minas Gerais, Brasil

Lia Raquel Moreira Oliveira Universidade do Minho, Portugal

Belmira Oliveira Bueno Universidade de São Paulo, Brasil

António Teodoro Universidade Lusófona, Portugal

Pia L. Wong California State University Sacramento, U.S.A

Sandra Regina Sales Universidade Federal Rural do Rio de Janeiro, Brasil

Elba Siqueira Sá Barreto_Fundação Carlos Chagas, Brasil

Manuela Terrasêca Universidade do Porto, Portugal

Robert Verhine Universidade Federal da Bahia, Brasil

Antônio A. S. Zuin Universidade Federal de São Carlos, Brasil 


\section{education policy analysis archives editorial board}

Editor Gustavo E. Fischman (Arizona State University)

Associate Editors: David R. Garcia (Arizona State University), Stephen Lawton (Arizona State University)

Rick Mintrop, (University of California, Berkeley) Jeanne M. Powers (Arizona State University)

Jessica Allen University of Colorado, Boulder

Gary Anderson New York University

Michael W. Apple University of Wisconsin, Madison

Angela Arzubiaga Arizona State University

David C. Berliner Arizona State University

Robert Bickel Marshall University

Henry Braun Boston College

Eric Camburn University of Wisconsin, Madison

Wendy C. Chi* University of Colorado, Boulder

Casey Cobb University of Connecticut

Arnold Danzig Arizona State University

Antonia Darder University of Illinois, UrbanaChampaign

Linda Darling-Hammond Stanford University

Chad d'Entremont Strategies for Children

John Diamond Harvard University

Tara Donahue Learning Point Associates

Sherman Dorn University of South Florida

Christopher Joseph Frey Bowling Green State University

Melissa Lynn Freeman* Adams State College

Amy Garrett Dikkers University of Minnesota

Gene V Glass Arizona State University

Ronald Glass University of California, Santa Cruz

Harvey Goldstein Bristol University

Jacob P. K. Gross Indiana University

Eric M. Haas WestEd

Kimberly Joy Howard* University of Southern California

Aimee Howley Ohio University

Craig Howley Ohio University

Steve Klees University of Maryland

Jaekyung Lee SUNY Buffalo
Christopher Lubienski University of Illinois, UrbanaChampaign

Sarah Lubienski University of Illinois, UrbanaChampaign

Samuel R. Lucas University of California, Berkeley

Maria Martinez-Coslo University of Texas, Arlington

William Mathis University of Colorado, Boulder

Tristan McCowan Institute of Education, London

Heinrich Mintrop University of California, Berkeley

Michele S. Moses University of Colorado, Boulder

Julianne Moss University of Melbourne

Sharon Nichols University of Texas, San Antonio

Noga O'Connor University of Iowa

João Paraskveva University of Massachusetts, Dartmouth

Laurence Parker University of Illinois, UrbanaChampaign

Susan L. Robertson Bristol University

John Rogers University of California, Los Angeles

A. G. Rud Purdue University

Felicia C. Sanders The Pennsylvania State University Janelle Scott University of California, Berkeley

Kimberly Scott Arizona State University

Dorothy Shipps Baruch College/CUNY

Maria Teresa Tatto Michigan State University

Larisa Warhol University of Connecticut

Cally Waite Social Science Research Council

John Weathers University of Colorado, Colorado Springs

Kevin Welner University of Colorado, Boulder

Ed Wiley University of Colorado, Boulder

Terrence G. Wiley Arizona State University

John Willinsky Stanford University

Kyo Yamashiro University of California, Los Angeles

* Members of the New Scholars Board 


\section{archivos analíticos de políticas educativas consejo editorial}

Editor: Gustavo E. Fischman (Arizona State University) Editores. Asociados Alejandro Canales (UNAM) y Jesús Romero Morante (Universidad de Cantabria)

Armando Alcántara Santuario Instituto de Investigaciones sobre la Universidad y la Educación, UNAM México

Claudio Almonacid Universidad Metropolitana de Ciencias de la Educación, Chile

Pilar Arnaiz Sánchez Universidad de Murcia, España

Xavier Besalú Costa Universitat de Girona, España Jose Joaquin Brunner Universidad Diego Portales, Chile

Damián Canales Sánchez Instituto Nacional para la Evaluación de la Educación, México

María Caridad García Universidad Católica del Norte, Chile

Raimundo Cuesta Fernández IES Fray Luis de León, España

Marco Antonio Delgado Fuentes Universidad Iberoamericana, México

Inés Dussel FLACSO, Argentina

Rafael Feito Alonso Universidad Complutense de Madrid, España

Pedro Flores Crespo Universidad Iberoamericana, México

Verónica García Martínez Universidad Juárez Autónoma de Tabasco, México

Francisco F. García Pérez Universidad de Sevilla, España

Edna Luna Serrano Universidad Autónoma de Baja California, México

Alma Maldonado Departamento de Investigaciones Educativas, Centro de Investigación y de Estudios Avanzados, México

Alejandro Márquez Jiménez Instituto de Investigaciones sobre la Universidad y la Educación, UNAM México

José Felipe Martínez Fernández University of California Los Angeles, USA
Fanni Muñoz Pontificia Universidad Católica de Perú

Imanol Ordorika Instituto de Investigaciones Economicas - UNAM, México

Maria Cristina Parra Sandoval Universidad de Zulia, Venezuela

Miguel A. Pereyra Universidad de Granada, España

Monica Pini Universidad Nacional de San Martín, Argentina

Paula Razquin UNESCO, Francia

Ignacio Rivas Flores Universidad de Málaga, España

Daniel Schugurensky Universidad de Toronto-Ontario Institute of Studies in Education, Canadá

Orlando Pulido Chaves Universidad Pedagógica Nacional, Colombia

José Gregorio Rodríguez Universidad Nacional de Colombia

Miriam Rodríguez Vargas Universidad Autónoma de Tamaulipas, México

Mario Rueda Beltrán Instituto de Investigaciones sobre la Universidad y la Educación, UNAM México

José Luis San Fabián Maroto Universidad de Oviedo, España

Yengny Marisol Silva Laya Universidad Iberoamericana, México

Aida Terrón Bañuelos Universidad de Oviedo, España

Jurjo Torres Santomé Universidad de la Coruña, España

Antoni Verger Planells University of Amsterdam, Holanda

Mario Yapu Universidad Para la Investigación Estratégica, Bolivia 